\title{
Quantitation of Contacts Among Sensory, Motor, and Serotonergic Neurons in the Pedal Ganglion of Aplysia
}

\author{
Han Zhang, Marcy Wainwright, John H. Byrne, and Leonard J. Cleary' \\ W.M. Keck Center for the Neurobiology of Learning and Memory, Department of Neurobiology and Anatomy, \\ University of Texas-Houston Medical School, Houston, Texas 77225, USA
}

\begin{abstract}
Present models of long-term sensitization in Aplysia californica indicate that the enhanced behavioral response is due, at least in part, to outgrowth of sensory neurons mediating defensive withdrawal reflexes. Presumably, this outgrowth strengthens pre-existing connections by formation of new synapses with follower neurons. However, the relationship between the number of sensorimotor contacts and the physiological strength of the connection has never been examined in intact ganglia. As a first step in addressing this issue, we used confocal microscopy to examine sites of contact between sensory and motor neurons in naive animals. Our results revealed relatively few contacts between physiologically connected cells. In addition, the number of contact sites was proportional to the amplitude of the EPSP elicited in the follower motor neuron by direct stimulation of the sensory neuron. This is the first time such a correlation has been observed in the central nervous system. Serotonin is the neurotransmitter most closely examined for its role in modulating synaptic strength at the sensorimotor synapse. However, the structural relationship of serotonergic processes and sensorimotor synapses has never been examined. Surprisingly, serotonergic processes usually made contact with sensory and motor neurons at sites located relatively distant from the sensorimotor synapse. This result implies that heterosynaptic regulation is due to nondirected release of serotonin into the neuropil.
\end{abstract}

Defensive withdrawal reflexes of the marine mollusk Aplysia californica are attractive model systems for the study of cellular and molecular plasticity underlying learning. The reflexes of tail-siphon withdrawal (Walters et al. 1983b; Scholz and Byrne 1987) and siphon-gill withdrawal (Byrne et al. 1974; Frost et al. 1985) have been studied in detail, and elements of the underlying neural circuits have been well characterized. Both reflexes are mediated by monosynaptic circuits composed of sensory and motor neurons, but polysynaptic circuits also contribute (Cleary et al. 1995).

Both withdrawal reflexes can be enhanced by sensitization, a form of nonassociative learning (Castellucci et al. 1970; Carew et al. 1971; Walters et al. 1983a). Long-term sensitization has been correlated with cellular changes in sensory neurons mediating these reflexes. These include both biophysical (Frost et al. 1985; Scholz and Byrne 1987; Cleary et al. 1998) and morphological changes (Bailey and Chen 1988a,b; Wainwright et al. 2002). Biophysical changes would presumably affect pre-existing synapses. On the other hand, outgrowth would presumably contribute to behavioral modification by forming new contacts with pre-existing follower neurons, or by recruiting new followers into the circuit.

Several modulatory transmitters have been shown to affect the strength of the sensorimotor circuit (Cleary et al. 1995). Many models indicate that these transmitters activate receptors at a site close to the sensorimotor synapse (Kandel and Schwartz 1982; Goelet et al. 1986; Byrne et al. 1990, 1991; Hawkins et al. 1993; Bailey et al. 2000; Kandel 2001; Marinesco and Carew 2002), although that specific issue has never been demonstrated.

\footnotetext{
${ }^{1}$ Corresponding author.

E-MAIL len.cleary@uth.tmc.edu; FAX (713) 500-0621.

Article and publication are at http://www.learnmem.org/cgi/doi/10.1101/ Im.63903.
}

Of the modulatory transmitters, serotonin is the best candidate to mediate the cellular affects of sensitization (Glanzman et al. 1989; Mackey et al. 1989; Wright et al. 1995; Marinesco and Carew 2002). Morphological evidence indicates that serotonergic neurons provide input to sensory neurons (Bailey et al. 1983; Kistler Jr. et al. 1985; Zhang et al. 1991). The structural proximity of serotonergic processes would enable elevated levels of intracellular second messengers and activated protein kinases to diffuse to nearby synapses and phosphorylate target proteins. The presumption is that axons containing serotonin would themselves make synapses close to the sensorimotor contacts, but this relationship has never been demonstrated (but see Bailey et al. 1983).

To investigate the connectivity of sensory, motor, and modulatory neurons, it is necessary to quantify the number of synaptic contacts in a relatively large volume of neuropil. Recently, confocal microscopy has been used for high resolution analysis of connections between labeled neurons (Gan et al. 1999; Cabirol-Pol et al. 2000; Jacoby et al. 2000; Hiesinger et al. 2001; Hammar and Maxwell 2002; Gray and Weeks 2003). We used this technique to identify sites of proximity between sensory neurons and their followers. We found that there were relatively few sites of contact, and that the number of contacts was proportional to the amplitude of the evoked EPSP. In addition, we observed a substantial number of serotonergic contacts with both sensory and motor neurons, but these contacts were not located close to the sensorimotor contacts.

\section{RESULTS}

Identification of Sites of Apposition Between Tail Sensory and Tail Motor Neurons

The tail sensory neurons are located on the ventral surface of the ventrocaudal sensory cluster (VC cluster) of the pleural ganglion 
(Fig. 1; Walters et al. 1983a; Zhang et al. 1993). The sensory neurons project through the pleural-pedal connective into the pedal ganglion, where they make monosynaptic contacts onto one or more tail motor neurons (Walters et al. 1983a). The motor neurons do not project back to the pleural ganglion (Fig. 1). Both sensory and motor neurons project to the tail through peripheral nerve P9 (Fig. 1; see also Walters et al. 1983a).

Putative sites of contact were examined in the pedal ganglion by intracellular dye injection in concert with confocal microscopy. The full extent of the sensory neuron arborization within the pedal ganglion was examined, but only microscopic fields containing both sensory and motor processes were scanned. We did not examine the pleural ganglion because there were no motor neuron processes. Also, we did not examine peripheral nerve $\mathrm{P} 9$ because of the variable extent to which sensory neuron processes in that nerve were filled. To begin, 2D maximum intensity projections were made through the entire volume (Fig. 2A), revealing relatively long contiguous axonal segments. These projections facilitated identification of potential sites of contact, which appeared yellow. To confirm that these were in fact contacts, a narrow volume of sections was constructed (Fig. 2B). Proximity of contacts was confirmed by rotating the pixel volumes (Fig. 2C). If the putative contact remained yellow throughout the rotation, it was scored as a contact. If a separation was observed during the rotation, it was not scored. Sites of contact are usually yellow in individual optical sections, indicating that the two processes are within the same voxel $(0.19$ $\mu \mathrm{m} \times 0.19 \mu \mathrm{m} \times 0.5 \mu \mathrm{m}$; Fig. 2D). The observer did not know the functional status of the connection (i.e., connected or unconnected as measured electrophysiologically) between labeled neurons (see below).

Relatively few contacts were observed between pairs of physiologically connected neurons $(24 \pm 4.2$, mean \pm SEM, $n=9$; Fig. 3A). Neurons that were not functionally coupled had essentially no contacts $(0.7 \pm 0.4, n=7)$. The difference between

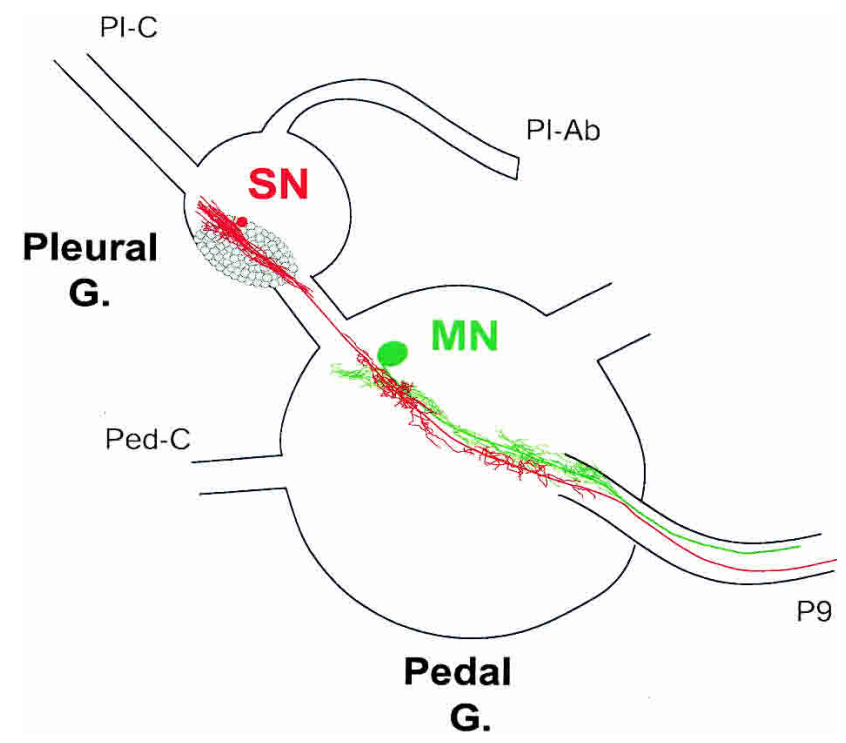

Figure 1 Schematic representation of tail sensory and motor neurons in the central nervous system of Aplysia. The somata of tail sensory neurons lie in the ventrocaudal cluster of the pleural ganglion. Sensory neurons, one of which is illustrated here (red), project through the pleural-pedal connective into the pedal ganglion. Monosynaptic contacts are formed with one or more tail motor neurons (green), whose cell bodies are located in the pedal ganglion. Both neurons project to the tail through peripheral nerve P9. The pleural abdominal ( $\mathrm{Pl}-\mathrm{Ab}$ ), pleural-cerebral ( $\mathrm{PI}$ $\mathrm{C})$, and pedal-cerebral (Ped-C) connectives are shown for orientation. physiologically connected and unconnected pairs of neurons was statistically significant $\left(t_{14}=4.94, p<0.001\right)$.

In physiologically connected neurons (Fig. 3B), the number of contact sites was positively correlated with the amplitude of the EPSP (Spearman $r=0.79, p<0.05, n=7$ ). This result implies that the number of contacts is a reasonable estimate of the number of synapses. Other methods, such as electron microscopy, will be necessary to confirm that all contacts are, in fact, sites of transmitter release.

\section{Proximity of Serotonergic Input to Sensorimotor Contacts}

Serotonin (5-HT) plays a major role in mediating sensitization of Aplysia withdrawal reflexes. Application of serotonin can mimic the effects of sensitization training (Kandel and Schwartz 1982; Walters et al. 1983b). Depletion of serotonin has been shown to block facilitation of the behavioral reflex (Glanzman et al. 1989). Moreover, sensitizing stimulation leads to release of serotonin (Levenson et al. 1999; Marinesco and Carew 2002). Previous studies using immunocytochemistry and electron microscopy demonstrated contacts between serotonergic processes and the cell body and processes of sensory neurons (Kistler Jr. et al. 1985; Zhang et al. 1991), but little is known regarding the relationship of serotonergic inputs to sensorimotor contacts.

Using confocal microscopy as described above, we examined the relationship of serotonergic axons to pairs of sensory and motor neurons. This required an additional step of immunofluorescence. To ensure full penetration of the antibody, thinner sections $(16 \mu \mathrm{m})$ were used. The neuropil of the pedal ganglion contains a large number of serotonin-immunoreactive neurites (Fig. 4A; Tritt et al. 1983; Ono and McCaman 1984; Nolen and Carew 1994; Wright et al. 1995; Marinesco and Carew 2002). As before, only sections with both sensory and motor neuron processes were examined. In addition, the arborizations of both sensory and motor neurons were screened for contacts by looking for additive color combinations. Sensorimotor combinations appeared yellow, as above. Serotonergic (blue)-sensory (red) contacts appeared magenta, and serotonergic (blue)-motor (green) contacts appeared cyan (Fig. 4).

In this experiment, there were $20 \pm 2$ contacts $(n=3)$ between sensory and motor neurons in the pedal ganglion. Within the regions analyzed (see Materials and Methods), there were more contacts between serotonergic and sensory neurons $(49.6 \pm 20, n=3)$ than between serotonergic and motor neurons $(24 \pm 7, n=3)$. There are substantially more motor neuron processes in the pedal ganglion than sensory neuron processes. Therefore, although the density of 5-HT-MN contacts is lower, the total number of 5-HT-MN contacts is greater than the number of 5-HT-SN contacts.

Surprisingly, serotonergic contacts were not located close to sensorimotor contacts. The straight-line distance (see Fig. 4A) between serotonergic contacts with sensory neurons (5-HT-SN) and sensorimotor contacts $(\mathrm{SN}-\mathrm{MN})$ was measured in projections. The closest $25 \%$ of the 5 -HT-SN contacts were within 9.6 $\mu \mathrm{m}$ (Fig. 5A). The closest contact was $3.6 \mu \mathrm{m}$ away. Of 60 sensorimotor contacts, nine were in sections that did not have a 5-HT$\mathrm{SN}$ contact. Therefore, the distance of the closest 5-HT-SN contact could not be determined for those sensorimotor contacts.

The distance between serotonergic contacts onto the motor neuron (5-HT-MN) and SN-MN contacts was also investigated. In the three preparations, again, the closest $25 \%$ of the $5-\mathrm{HT}-\mathrm{MN}$ contacts were within $9.6 \mu \mathrm{m}$ (Fig. 5B), with the closest being 4.2 $\mu \mathrm{m}$. Of 60 sensorimotor contacts, 20 were in sections that did not have a 5-HT-MN contact, and the closest contact was not determined.

On the other hand, there are numerous serotonergic processes coursing through the neuropil in the vicinity of SN-MN 

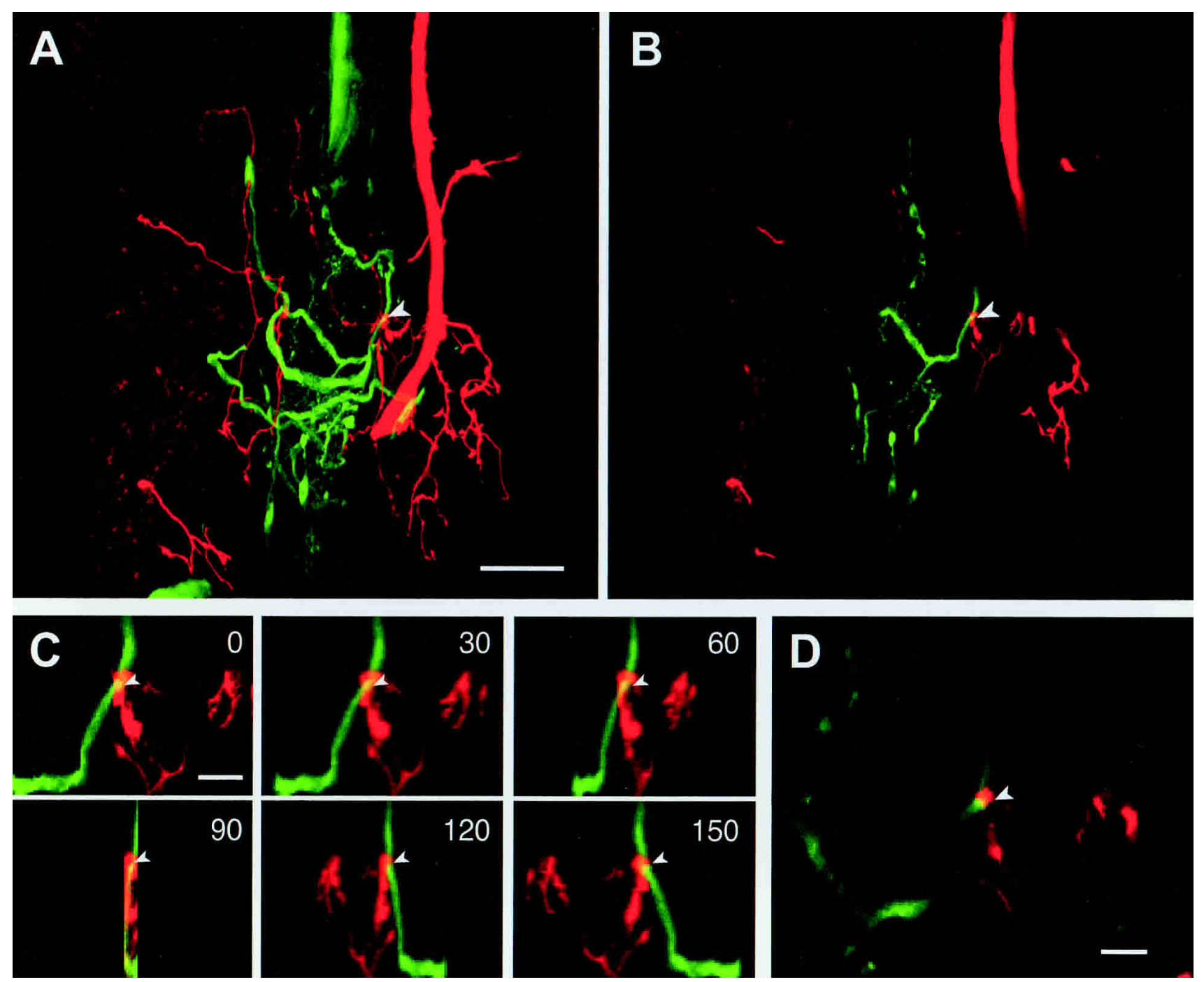

Figure 2 Identification of potential sites of contact between tail sensory and motor neurons. $(A)$ Confocal image through a region of the pedal ganglion containing branches of both the sensory neuron (red) and the motor neuron (green). The image was calculated by projecting through a stack of 43 optical sections. Although there are numerous branches from both neurons, relatively few potential contacts (yellow) were observed. One of these (arrowhead) was examined more closely in subsequent panels. Scale bar, $20 \mu \mathrm{m}$. (B) Contacts are more reliably observed by analyzing a subset of the data. This image was calculated by projecting through six optical sections surrounding the site of a potential contact (arrowhead). The scale is the same as $A$. (C) A small volume surrounding the potential contact from the image stack in $B$ (six optical sections) was then rotated around the vertical axis. Examples from $0^{\circ}, 30^{\circ}, 60^{\circ}, 90^{\circ}, 120^{\circ}$, and $150^{\circ}$ angles are shown. The yellow zone of contact is observed in all panels, confirming proximity. Scale bar in the 0 degree projection, $4 \mu \mathrm{m}$. Because the voxels are rectangular (see Materials and Methods), the scale bar corresponds to $10 \mu \mathrm{m}$ for the $90^{\circ}$ projection. (D) A single optical section from the stack illustrated in $A$. In this case, the site of contact (arrowhead) is still observed as yellow pixels. Scale bar, $4 \mu \mathrm{m}$.

contacts. These are generally much closer than the serotonergic contacts (Fig. 5C). The closest $25 \%$ were within $2.3 \mu \mathrm{m}$, with the closest being $0.8 \mu \mathrm{m}$. Serotonergic processes were observed in all sections that contained sensorimotor contacts. Because serotonergic processes tend to pass closer to sensorimotor contacts, it is likely that they play a role in modulating synaptic transmission. Therefore, these data indicate that modulatory stimuli cause release of 5-HT into the neuropil from nondirected synapses (see also Marinesco and Carew 2002). The transmitter would then diffuse to receptors on the sensory neuron that are close enough to sensorimotor contacts to modulate transmitter release.

\section{DISCUSSION}

Sensorimotor synapses in Aplysia have been studied in great detail for many years. In electrophysiological recordings, stimula- tion of a sensory neuron evokes a unitary EPSP in a follower motor neuron. The unitary EPSP is undoubtedly the summed response to release of transmitter from multiple active zones. This report is the first attempt to correlate the number of contacts between sensory and motor neurons with EPSP amplitude in intact ganglia.

On average, we found 24 contacts per pair of neurons. The number of contacts was significantly correlated with the size of the EPSP. The slope of the linear regression line was $264 \mu \mathrm{V} /$ contact when the line was forced to include the point $(0,0)$. A similar analysis of cultured sensory and motor neurons also demonstrated a linear relationship between the number of contacts and EPSP amplitude (Schacher and Montarolo 1991). In that experiment, the slope of the regression line was $\sim 660 \mu \mathrm{V} /$ contact. This result indicates that individual contacts are stronger in cul- 
ture than in the ganglion. The larger value can be attributed in part to the fact that motor neurons were hyperpolarized by 30 $\mathrm{mV}$ from the resting potential, whereas in the present study, the motor neuron was maintained at the resting potential. Moreover, the sensory neuron was cultured with L7, which is from the abdominal ganglion and may have a different postsynaptic sensitivity than tail motor neurons. Additional experiments will be required to identify other factors contributing to the relative strength of synapses in culture.

The estimated contribution of a single contact to the EPSP is an upper limit, because we may not have counted all contacts. For example, we did not examine the peripheral nerve. Both sensory and motor neurons project to the body wall through pedal nerve $\mathrm{P} 9$, and synapses could, in principle, form there as well. In addition, we required an observable varicosity in the sensory process, and synapses occurring at an intervaricose segment would not have been counted.

The contribution of a contact to the EPSP calculated above is probably not the same as the miniature EPSP observed in quantal analysis. Such an analysis has not been performed for the tail sensorimotor synapse. In one study of siphon sensory and motor neurons, however, miniature EPSPs were $30 \mu \mathrm{V}$ with an average evoked EPSP amplitude of $247 \mu \mathrm{V}$ (Castellucci and Kandel 1974). The latter value could be affected by the bathing medium, which was optimized for low transmitter release. If the miniature EPSP did represent a single contact, however, then there were would be roughly eight contacts between the sensory and motor neurons in that circuit. This number is lower than what we observed at the tail sensorimotor synapse, but is roughly within the same range.

Quantal analysis of release from pleural sensory neurons has been examined, but only in isolated cell culture. When cultured with the siphon motor neuron L7, pleural sensory neurons appear to have a quantal size of $60-100 \mu \mathrm{V}$ (Dale et al. 1988). This range of values is lower than the value of $\sim 660 \mu \mathrm{V} /$ contact observed in similar cultures discussed above (Schacher and Montarolo 1991). Again, this may be explained in part by hyperpolarization of the postsynaptic cell in the latter study. On the other hand, it is possible that quantal size does not represent the full release capacity of a single contact. This could be caused by the presence of multiple active zones per contact (Bailey et al. 1979).

It is important to note that a higher-resolution technique,
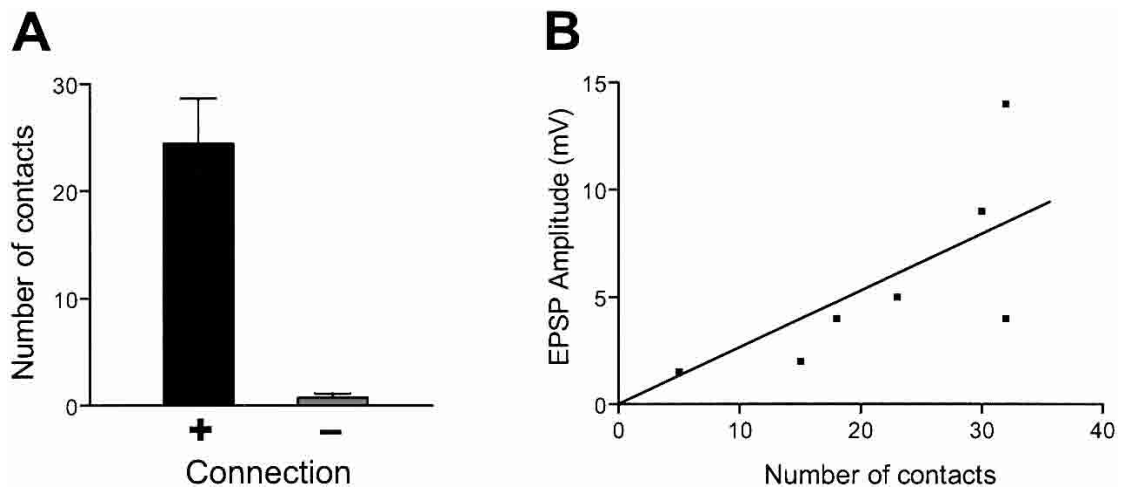

Figure 3 Quantification of contacts between tail sensory neurons and follower motor neurons. (A) The total number of contact sites were counted from serial sections through the extent of the pedal ganglion. More contacts were found between pairs of physiologically connected neurons (+) than between neurons that were not connected $(-; 24 \pm 4.2$ vs. $0.7 \pm 0.4$; Mean \pm SEM). This difference was statistically significant $\left(t_{14}=4.94, p<0.001\right)$. (B) In physiologically connected neurons, the number of contact sites was proportional to the amplitude of the EPSP (Spearman $r=0.79, p<0.05, n=7)$. This result indicates that the number of contacts is a reasonable estimate of the number of synapses, even if the presence of an active zone cannot be confirmed at each contact. The regression line was constrained to pass through $(0,0)$. such as electron microscopy, would not have added additional information to this initial study. The number of contacts we observed was roughly comparable to that expected from the size of the contacts were expected between cells that were not physiologically connected, and essentially none were found. Therefore, the resolucontacts with the expectation of observing direct apposition of pre- and postsynaptic membranes and the presence of

The Structural Proximity of Serotonergic Neurons, Sensory Neurons, and Motor Neurons

The structural relationship between neurons containing modulatory transmitters and the synapses that they affect has never been studied. By adding an immunofluorescence step, we examined directly the proximity of serotonergic processes to sensorimotor contacts. In three preparations, we did not sory neuron or a motor neuron in the proximity of a sensorimotor contact. Indeed, the closest contact was a straight-line distance of $\sim 4 \mu \mathrm{m}$. The intraneuronal distance was presumably longer, because processes generally did not project along the section $(16 \mu \mathrm{m})$, and therefore, we could not rule out the possibility that contacts in adjacent sections were closer than $4 \mu \mathrm{m}$. Nevertheless, out of 60 sensorimotor contacts, we should have seen a few serotonergic contacts in close proximity if they were common.

Serotonin exerts its modulatory effects through receptors that are coupled to G proteins (Li et al. 1995; Angers et al. 1998). Therefore, compared with ligand-gated receptors such as those at the sensorimotor synapse, they are likely to act over relatively messengers. Nevertheless, the distance of serotonergic input from sensorimotor synapse profiles that we measured begs the question of how effective those contacts are in elevating levels of intracellular second messengers, such as cAMP, at the synapse. Our hypothesis is that serotonin is released into the extracellular space to bind with receptors near the synaptic terminal (Marinesco and Carew 2002).

Very few neurons in Aplysia have been identified that, when stimulated, enhance the strength of sensorimotor synapses. Examples include the serotonergic neuron CB1 (Mackey et al. 1989; Wright et al. 1995) and L29, whose transmitter type has not been identified (Hawkins and Schacher 1989). Many more such neurons must exist. Release from nondirected synapses could explain why they have not yet been readily identified. Stimulation of a single neuron would probably not by itself be sufficient to raise the concentration of extracellular transmitter to effective levels.

If serotonin is released from nondirected synapses, then what is the function of contacts between serotonergic and sensory neurons? One possibility is that they are not necessary for modulating the sensorimotor synapse, raising the interesting possibility that sensory neurons are presynaptic elements at sites of contact with serotonergic processes. Therefore, these contacts 

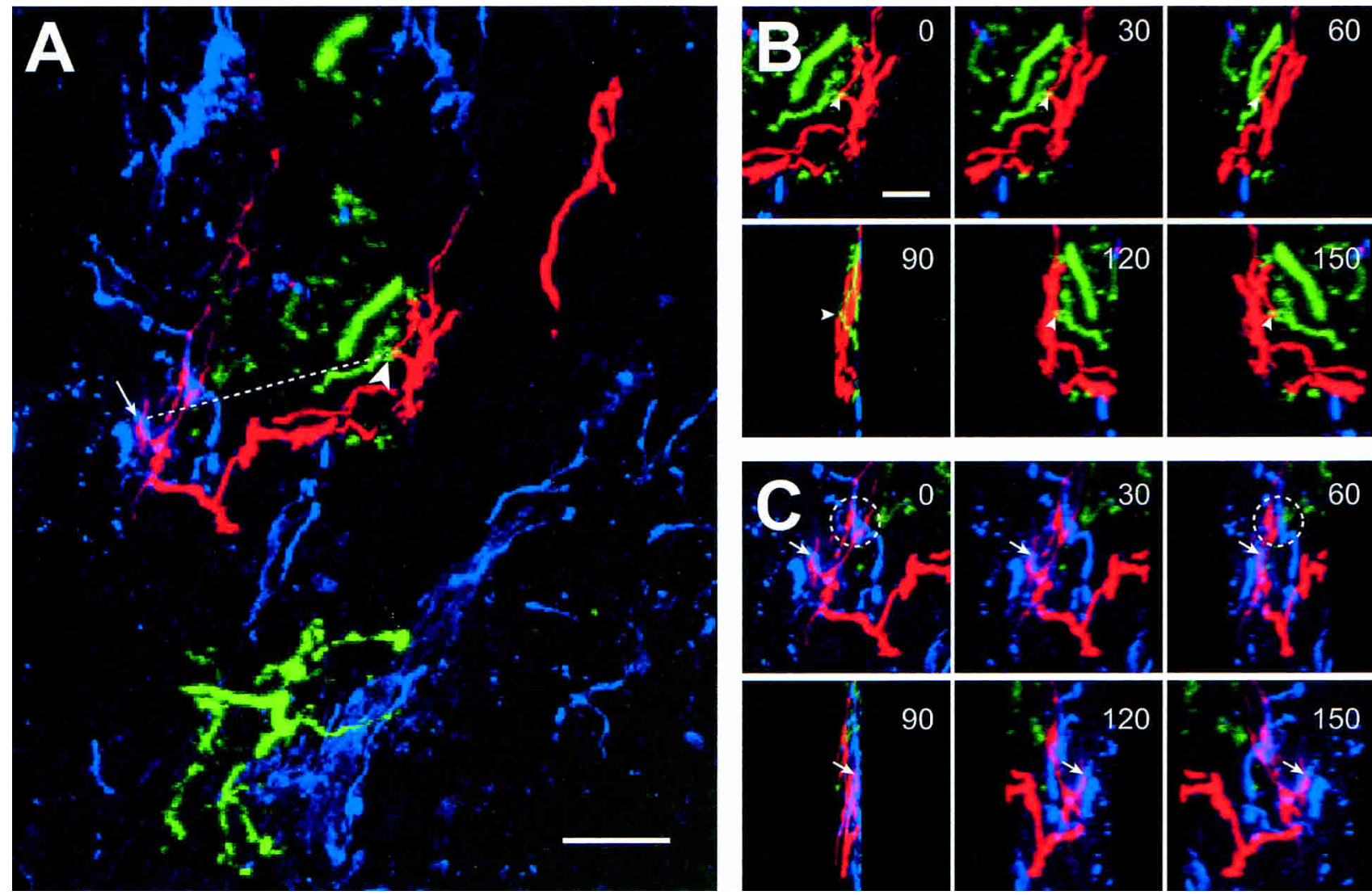

Figure 4 Localization of serotonergic contacts with sensory and motor neurons. (A) Sections with labeled sensory and motor neurons were stained with antibody directed against 5-HT, the presumed modulatory transmitter of the sensorimotor synapse. This image is a projection through 16 optical sections. As in Figure 2, contacts between sensory (red) and motor (green) neurons appear yellow (large arrowhead). Serotonergic fibers appear blue. In the projection, apparent contacts between sensory neurons and 5-HT-immunoreactive processes appear magenta (arrow). Contacts between motor neurons and 5-HT immunoreactive processes appear cyan. In this print, magenta and cyan may be difficult to distinguish from red and green, respectively. The straight-line distance between the sensorimotor contact and the nearest serotonergic-sensory contact is indicated by the dashed line, and measures $25 \mu \mathrm{m}$ in this case. Scale bar, $10 \mu \mathrm{m}$. (B) A subset of the image stack shown in $A$ (16 optical sections) was examined by rotation around the vertical axis. The contact between the sensory and motor neuron (arrowhead) was indicated by presence of overlap (yellow pixels, arrowhead) from all directions, confirming that the two points are, indeed, in close proximity. Examples from images rotated $0^{\circ}, 30^{\circ}, 60^{\circ}, 90^{\circ}, 120^{\circ}$, and $150^{\circ}$ are shown. Scale bar in the 0 degree projection, $5 \mu \mathrm{m}$. Because the voxels are rectangular (see Materials and Methods), the scale bar corresponds to $12.5 \mu \mathrm{m}$ for the $90^{\circ}$ projection. (C) The contact between serotonergic and sensory neurites (arrow) in $A$ was also examined following rotation. Note that in this field, there is another apparent contact (dashed line), but on rotation the neurites were clearly not in proximity. Same scale as $B$.

may represent sites at which afferent information is conducted to modulatory pathways. For the same reasons mentioned above, it would be unlikely that stimulation of a single sensory neuron could release an amount of serotonin sufficient to modulate the sensorimotor synapse.

Compared with electron microscopy, confocal microscopy is an efficient way to examine relatively large volumes of tissue in large numbers of animals. In this paper, we demonstrated that confocal microscopy is also a valid means to examine synaptic strength between identified neurons. In a previous study, we demonstrated that long-term sensitization training produces neurite outgrowth and formation of new varicosities (Wainwright et al. 2002). We expect confocal microscopy to be a useful tool for assessing the functional significance of these structural modifications.

\section{MATERIALS AND METHODS}

\section{Identification of Physiologically Connected Cells}

Aplysia californica (150-350 g) were anesthetized by injection of isotonic $\mathrm{MgCl}_{2}(500 \mathrm{~mL} / \mathrm{kg})$, and the pleural-pedal ganglia were removed. The connective tissue sheath was surgically removed to expose the tail sensory and motor neurons. Intracellular recordings were made through glass microelectrodes with a resistance of 5-15 $\mathrm{M} \Omega$. To reduce spontaneous activity and the possibility of eliciting polysynaptic input to motor neurons by stimulating sensory neurons, the artificial seawater contained three times the normal concentrations of $\mathrm{Mg}^{2+}$ and $\mathrm{Ca}^{2+}$ (Byrne et al. 1978). Tail motor neurons were initially identified by size, shape, and location along the fiber tract in the anterior pedal ganglion arising from the pleural-pedal connective (Fig. 1). Additional electrophysiological characteristics were also examined, including the antidromic action potential elicited by stimulation of peripheral nerve P9 (Walters et al. 1983a). A sensory neuron and a follower motor neuron were impaled and, after a brief period, both cells achieved a stable resting potential. Depolarizing current pulses of increasing intensity ( $0.1 \mathrm{nA}$ steps, $50 \mathrm{msec}$ duration) were then applied to the sensory neuron until the cell fired. In connected pairs of cells, an EPSP was evoked in the motor neuron.

\section{Labeling of Cells and Tissue Processing}

After determination of a physiological connection, dextran-conjugated tetramethylrhodamine $(3000 \mathrm{MW}, 2 \%$ in $0.9 \% \mathrm{KCl}$; Molecular Probes) was injected by pressure into sensory neurons and dextran-conjugated Alexa 488 (10,000 MW, 2\% in 0.9\% KCl; Mo- 


\section{A. Closest $5 \mathrm{HT}-\mathrm{SN}$ contact}

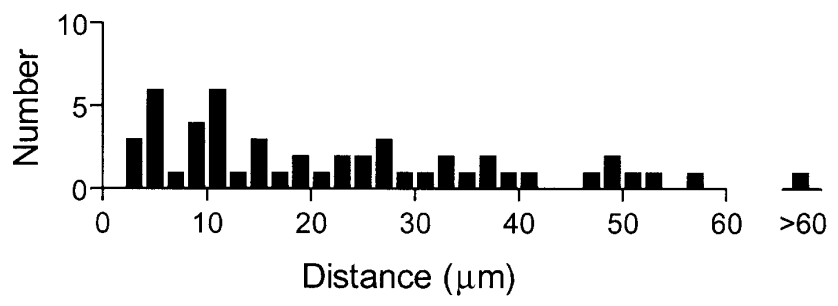

B. Closest $5 \mathrm{HT}-\mathrm{MN}$ contact

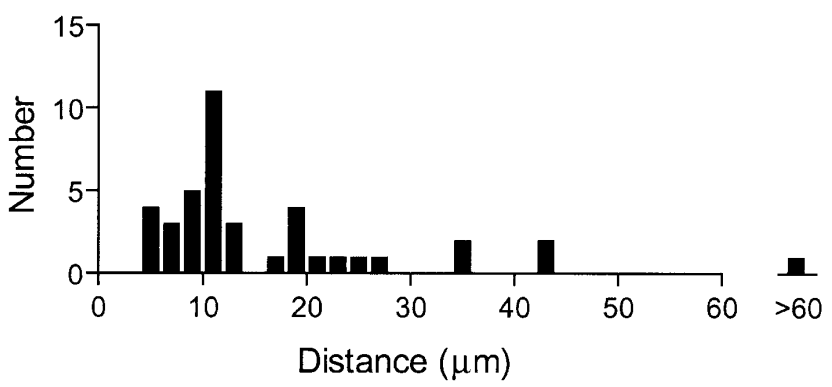

C. Closest 5-HT process

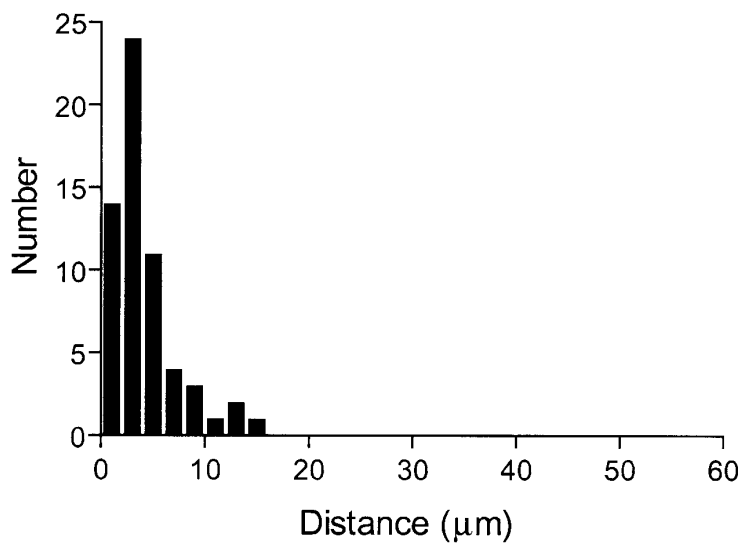

Figure 5 Serotonergic inputs were not in close proximity to SN-MN contacts. $(A)$ The straight-line distance between a sensorimotor contact and the closest serotonergic-sensory neuron (5-HT-SN) contact. (B) The straight-line distance between a sensorimotor contact and the closest serotonergic-motor neuron (5-HT-MN) contact. (C) The straight-line distance between a sensorimotor contact and the closest serotonergic process.

lecular Probes) was injected into motor neurons. In some experiments, the sensory neuron was not injected. Instead, a neighboring sensory neuron was identified that did not elicit an EPSP in the motor neuron. That sensory neuron was injected as above. Ganglia were then placed in a $15^{\circ} \mathrm{C}$ incubator for $4 \mathrm{~h}$ to allow diffusion of the dye throughout the neuronal arborization. Following incubation, ganglia were fixed in paraformaldehyde (4\% in phosphate-buffered saline plus 30\% sucrose) and cryosectioned at a nominal thickness of $40 \mu \mathrm{m}$. Slides were mounted in an antifade medium (ProLong; Molecular Probes) to minimize photobleaching during microscopic examination.

Immunohistochemistry for Labeling of Serotonergic Fibers

In some experiments, immunofluorescence was used as a third label. All ganglia were incubated overnight in PBS following fixation. Subsequently, ganglia were sectioned on a cryostat at a nominal thickness of $16 \mu \mathrm{m}$. Slides were rinsed in PBS containing $0.1 \%$ Triton X-100 and permeabilized by serial dehydration/rehydration through an ethanol series. Sections were treated with $0.3 \%$ hydrogen peroxide in PBS for 10 min to reduce autofluorescence. Nonspecific binding was blocked by incubating the sections in $2 \%$ normal rabbit serum (Vector Laboratories) at room temperature for $2 \mathrm{~h}$. Sections were then incubated overnight at $4^{\circ} \mathrm{C}$ in rabbit anti-serotonin antibody (DiaSorin) diluted (1:5000) in PBS containing $0.1 \%$ Triton X-100. Sections were then incubated in cy5-conjugated goat anti-rabbit antisera (1:100; Jackson Immunoresearch Laboratories) for $30 \mathrm{~min}$. Slides were coverslipped with the antifade mounting medium.

\section{Morphological Analysis}

All sections were imaged with a scanning confocal microscope (Bio-Rad, MRC 1024ES) under a $60 \times$ oil immersion lens (NA 1.4). Pixel noise was reduced by Kalman averaging over three scans. The zoom setting was 1 , and the aperture setting was 2.2 . The image size was set at $1024 \times 768$. Under these conditions, one pixel represents a square of $0.19 \mu \mathrm{m}$ on each side. The estimated thickness of an optical section was $0.5 \mu \mathrm{m}$. A stack of images was collected through the section with a stepsize of $0.5 \mu \mathrm{m}$. During image collection, the gain and black level values were adjusted to use the full range of pixel intensities (0-255) with very little saturation at either end of the intensity range. Images were collected in two passes to minimize bleeding of emission from Alexa into the tetramethylrhodamine channel. Alexa 488 was excited with a band pass filter that transmitted the 488-nm line of the $\mathrm{Kr}-\mathrm{Ar}$ laser, and tetramethylrhodamine was excited with a band pass filter that transmitted the 568-nm line. For triple labeling experiments, a third pass was added that excited Cy5 with the $647-\mathrm{nm}$ line.

Stacks of images were analyzed with MetaMorph Basic (Universal Imaging, Inc.). No additional image processing steps were used. Initial projections were screened for potential sites of contact by searching for the additive color combination. For example, red and green labels in close proximity appeared yellow. The data set was reduced to a small volume surrounding the potential contact and rotated to be sure that the two labeled processes remained in proximity. For the purpose of preparing Figures 2 and 4, image gain and gamma was adjusted in Photoshop (Adobe Systems, Inc.). The observer did not know the functional status of the connection (i.e., connected or unconnected as measured electrophysiologically) between labeled neurons.

In this study, each microscopic field was rapidly prescreened under manual control. Only volumes that contained processes from both sensory and motor neurons were fully scanned and saved for analysis. Contacts between sensory and motor neurons were counted only if the presynaptic component was a varicosity. The straight-line distance between serotonergic-sensory contacts and the nearest sensorimotor contacts was measured. This distance likely underestimates the shortest intra-axonal distance, because of the complex geometry of the processes.

\section{ACKNOWLEDGMENTS}

The authors thank Jennifer DeLeon for excellent technical assistance and Matthew Landman for his contribution to pilot experiments as a participant in the Undergraduate Summer Research Program. The authors also thank David Marshak and Evangelos Antzoulatos for their helpful comments on an earlier version of the manuscript. This work was supported by NIH grants T32 NS07373 and F31 MH12176 (M.L.W.), NS19895 (J.H.B.), and NS38100 (L.J.C.). 
The publication costs of this article were defrayed in part by payment of page charges. This article must therefore be hereby marked "advertisement" in accordance with 18 USC section 1734 solely to indicate this fact.

\section{REFERENCES}

Angers, A., Storozhuk, M.V., Duchaine, T., Castellucci, V.F., and DesGroseillers, L. 1998. Cloning and functional expression of an Aplysia 5-HT receptor negatively coupled to adenylate cyclase. J. Neurosci. 18: 5586-5593.

Bailey, C.H. and Chen, M. 1983. Morphological basis of long-term habituation and sensitization in Aplysia. Science 220: 91-93. . 1988a. Long-term memory in Aplysia modulates the total number of varicosities of single identified sensory neurons. Proc. Natl. Acad. Sci. 85: 2373-2377.

. 1988b. Long-term sensitization in Aplysia increases the number of presynaptic contacts onto the identified gill motor neuron L7. Proc. Natl. Acad. Sci. 85: 9356-9359.

Bailey, C.H., Thompson, E.B., Castellucci, V.F., and Kandel, E.R. 1979. Ultrastructure of the synapses of sensory neurons that mediate the gill-withdrawal reflex in Aplysia. J. Neurocytol. 8: 415-444.

Bailey, C.H., Hawkins, R.D., and Chen, M.C. 1983. Uptake of $\left[{ }^{3} \mathrm{H}\right]$ serotonin in the abdominal ganglion of Aplysia californica. Further studies on the morphological and biochemical basis of presynaptic facilitation. Brain Res. 272: 71-81.

Bailey, C.H., Giustetto, M., Huang, Y.Y., Hawkins, R.D., and Kandel, E.R. 2000. Is heterosynaptic modulation essential for stabilizing Hebbian plasticity and memory? Nat. Rev. Neurosci. 1: 11-20.

Byrne, J., Castellucci, V., and Kandel, E.R. 1974. Receptive fields and response properties of mechanoreceptor neurons innervating siphon skin and mantle shelf in Aplysia. J. Neurophysiol. 37: 1041-1064.

. 1978. Contribution of individual mechanoreceptor sensory neurons to defensive gill-withdrawal reflex in Aplysia. J. Neurophysiol. 41: $418-431$

Byrne, J.H., Baxter, D.A., Buonomano, D.V., and Raymond, J.L. 1990 Neuronal and network determinants of simple and higher-order features of associative learning: Experimental and modeling approaches. Cold Spring Harb. Symp. Quant. Biol. 55: 175-186.

Byrne, J.H., Baxter, D.A., Buonomano, D.V., Cleary, L.J., Eskin, A., Goldsmith, J.R., McClendon, E., Nazif, F.A., Noel, F., and Scholz, K.P. 1991. Neural and molecular bases of nonassociative and associative learning in Aplysia. Ann. NY Acad. Sci. 627: 124-149.

Cabirol-Pol, M.J., Mizrahi, A., Simmers, J., and Meyrand, P. 2000. Combining laser scanning confocal microscopy and electron microscopy to determine sites of synaptic contact between two identified neurons. J. Neurosci. Methods 97: 175-181.

Carew, T.J., Castellucci, V.F., and Kandel, E.R. 1971. An analysis of dishabituation and sensitization of the gill-withdrawal reflex in Aplysia. Int. J. Neurosci. 2: 79-98.

Castellucci, V.F. and Kandel, E.R. 1974. A quantal analysis of the synaptic depression underlying habituation of the gill-withdrawal reflex in Aplysia. Proc. Natl. Acad. Sci. 71: 5004-5008.

Castellucci, V., Pinsker, H., Kupfermann, I., and Kandel, E.R. 1970. Neuronal mechanisms of habituation and dishabituation of the gill-withdrawal reflex in Aplysia. Science 167: 1745-1748.

Cleary, L.J., Byrne, J.H., and Frost, W.N. 1995. Role of interneurons in defensive withdrawal reflexes in Aplysia. Learn. Mem. 2: 133-151.

Cleary, L.J., Lee, W.L., and Byrne, J.H. 1998. Cellular correlates of long-term sensitization in Aplysia. J. Neurosci. 18: 5988-5998.

Dale, N., Schacher, S., and Kandel, E.R. 1988. Long-term facilitation in Aplysia involves increase in transmitter release. Science 239: 282-285.

Frost, W.N., Castellucci, V.F., Hawkins, R.D., and Kandel, E.R. 1985. Monosynaptic connections made by the sensory neurons of the gilland siphon-withdrawal reflex in Aplysia participate in the storage of long-term memory for sensitization. Proc. Natl. Acad. Sci. 82: $8266-8269$.

Gan, W.B., Bishop, D.L., Turney, S.G., and Lichtman, J.W. 1999. Vital imaging and ultrastructural analysis of individual axon terminals labeled by iontophoretic application of lipophilic dye. J. Neurosci. Methods 93: 13-20.

Glanzman, D.L., Mackey, S.L., Hawkins, R.D., Dyke, A.M., Lloyd, P.E. and Kandel, E.R. 1989. Depletion of serotonin in the nervous system of Aplysia reduces the behavioral enhancement of gill withdrawal as well as the heterosynaptic facilitation produced by tail shock. $J$. Neurosci. 9: 4200-4213.

Goelet, P., Castellucci, V.F., Schacher, S., and Kandel, E.R. 1986. The long and the short of long-term memory-A molecular framework. Nature 322: 419-422.

Gray, J.R. and Weeks, J.C. 2003. Steroid-induced dendritic regression reduces anatomical contacts between neurons during synaptic weakening and the developmental loss of a behavior. J. Neurosci. 23: $1406-1415$.

Hammar, I. and Maxwell, D.J. 2002. Serotoninergic and noradrenergic axons make contacts with neurons of the ventral spinocerebellar tract in the cat. J. Comp. Neurol. 443: 310-319.

Hawkins, R.D. and Schacher, S. 1989. Identified facilitator neurons L29 and L28 are excited by cutaneous stimuli used in dishabituation, sensitization, and classical conditioning of Aplysia. J. Neurosci. 9: $4236-4245$.

Hawkins, R.D., Kandel, E.R., and Siegelbaum, S.A. 1993. Learning to modulate transmitter release: Themes and variations in synaptic plasticity. Annu. Rev. Neurosci. 16: 625-665.

Hiesinger, P.R., Scholz, M., Meinertzhagen, I.A., Fischbach, K.F., and Obermayer, K. 2001. Visualization of synaptic markers in the optic neuropils of Drosophila using a new constrained deconvolution method. J. Comp. Neurol. 429: 277-288.

Jacoby, R.A., Wiechmann, A.F., Amara, S.G., Leighton, B.H., and Marshak, D.W. 2000. Diffuse bipolar cells provide input to OFF parasol ganglion cells in the macaque retina. J. Comp. Neurol. 416: $6-18$.

Kandel, E.R. 2001. The molecular biology of memory storage: A dialogue between genes and synapses. Science 294: 1030-1038.

Kandel, E.R. and Schwartz, J.H. 1982. Molecular biology of learning: Modulation of transmitter release. Science 218: 433-443.

Kistler Jr., H.B., Hawkins, R.D., Koester, J., Steinbusch, H.W., Kandel, E.R., and Schwartz, J.H. 1985. Distribution of serotonin-immunoreactive cell bodies and processes in the abdominal ganglion of mature Aplysia. J. Neurosci. 5: 72-80.

Levenson, J., Byrne, J.H., and Eskin, A. 1999. Levels of serotonin in the hemolymph of Aplysia are modulated by light/dark cycles and sensitization training. J. Neurosci. 19: 8094-8103.

Li, X.C., Giot, J.F., Kuhl, D., Hen, R., and Kandel, E.R. 1995. Cloning and characterization of two related serotonergic receptors from the brain and the reproductive system of Aplysia that activate phospholipase C. J. Neurosci. 15: 7585-7591.

Mackey, S.L., Kandel, E.R., and Hawkins, R.D. 1989. Identified serotonergic neurons LCB1 and RCB1 in the cerebral ganglia of Aplysia produce presynaptic facilitation of siphon sensory neurons. $J$. Neurosci. 9: 4227-4235

Marinesco, S. and Carew, T.J. 2002. Serotonin release evoked by tail nerve stimulation in the CNS of aplysia: Characterization and relationship to heterosynaptic plasticity. J. Neurosci. 22: 2299-2312.

Nolen, T.G. and Carew, T.J. 1994. Ontogeny of serotonin-immunoreactive neurons in juvenile Aplysia californica: Implications for the development of learning. Behav. Neural Biol. 61: 282-295.

Ono, J.K. and McCaman, R.E. 1984. Immunocytochemical localization and direct assays of serotonin-containing neurons in Aplysia. Neuroscience 11: 549-560.

Schacher, S. and Montarolo, P.G. 1991. Target-dependent structural changes in sensory neurons of Aplysia accompany long-term heterosynaptic inhibition. Neuron 6: 679-690.

Scholz, K.P. and Byrne, J.H. 1987. Long-term sensitization in Aplysia: Biophysical correlates in tail sensory neurons. Science 235: 685-687.

Tritt, S.H., Lowe, I.P., and Byrne, J.H. 1983. A modification of the glyoxylic acid induced histofluorescence technique for demonstration of catecholamines and serotonin in tissues of Aplysia californica. Brain Res. 259: 159-162.

Wainwright, M.L., Zhang, H., Byrne, J.H., and Cleary, L.J. 2002. Localized neuronal outgrowth induced by long-term sensitization training in Aplysia. J. Neurosci. 22: 4132-4141.

Walters, E.T., Byrne, J.H., Carew, T.J., and Kandel, E.R. 1983a. Mechanoafferent neurons innervating tail of Aplysia. I. Response properties and synaptic connections. J. Neurophysiol. 50: 1522-1542. . 1983b. Mechanoafferent neurons innervating tail of Aplysia. II. Modulation by sensitizing stimulation. J. Neurophysiol. 50: $1543-1559$.

Wright, W.G., Jones, K., Sharp, P., and Maynard, B. 1995. Widespread anatomical projections of the serotonergic modulatory neuron, CB1, in Aplysia. Invert. Neurosci. 1: 173-183.

Zhang, H., Byrne, J.H., and Cleary, L.J. 1993. Topographical organization of sensory neurons in the ventrocaudal cluster of the pleural ganglion of Aplysia. Soc. Neuroci. Abstr. 19: 813.

Zhang, Z.S., Fang, B., Marshak, D.W., Byrne, J.H., and Cleary, L.J. 1991. Serotoninergic varicosities make synaptic contacts with pleural sensory neurons of Aplysia. J. Comp. Neurol. 311: 259-270.

Received May 30, 2003; accepted in revised form August 18, 2003.
Learning \& Memory www.learnmem.org 


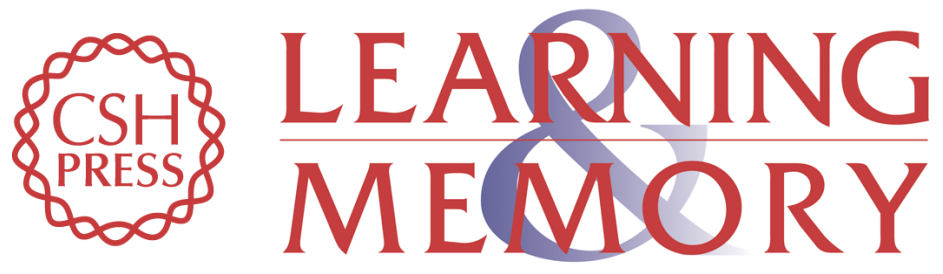

\section{Quantitation of Contacts Among Sensory, Motor, and Serotonergic Neurons in the Pedal Ganglion of Aplysia}

Han Zhang, Marcy Wainwright, John H. Byrne, et al.

Learn. Mem. 2003, 10:

Access the most recent version at doi:10.1101//m.63903

References This article cites 46 articles, 23 of which can be accessed free at:

http://learnmem.cshlp.org/content/10/5/387.full.html\#ref-list-1

License

Email Alerting Receive free email alerts when new articles cite this article - sign up in the box at the Service top right corner of the article or click here. 